Case Report

\title{
Renal Replacement Lipomatosis Presenting in the Setting of Ureteral Stricture with Absence of Renal Calculus Disease
}

\author{
Katrina Collins $\mathbb{D}^{1},{ }^{1}$ Eric Brocken, ${ }^{1}$ Laura M. Warmke, ${ }^{1}$ Temel Tirkes, ${ }^{2}$ and Michael Hwang ${ }^{1}$ \\ ${ }^{1}$ Department of Pathology, Indiana University, Indianapolis, IN 46202, USA \\ ${ }^{2}$ Department of Radiology and Clinical Sciences, Indiana University, Indianapolis, IN 46202, USA
}

Correspondence should be addressed to Katrina Collins; katcoll@iu.edu

Received 3 July 2021; Accepted 7 October 2021; Published 23 October 2021

Academic Editor: Yoji Nagashima

Copyright (c) 2021 Katrina Collins et al. This is an open access article distributed under the Creative Commons Attribution License, which permits unrestricted use, distribution, and reproduction in any medium, provided the original work is properly cited.

\begin{abstract}
Renal replacement lipomatosis of the kidney is a rare, benign entity in which extensive fibrofatty proliferation of the renal sinus is associated with marked atrophy of the renal parenchyma. It is often associated with calculi or long-standing inflammation. This entity may be confused with a fatty neoplasm of the kidney. A 51-year-old woman with a past medical history of pancreas transplant for type 1 diabetes subsequently developed ureteral stricture. This was initially managed by a nephrostomy tube and nephroureterostomy stenting with periodic exchanges to help restore urine flow; however, the renal function of the kidney progressively declined with recurrent and complicated urinary tract infections. She presented for kidney transplant with right native nephrectomy. Gross examination of the right kidney revealed a $12.8 \mathrm{~cm}$ renal sinus lipomatous mass replacing much of the kidney. Microscopically, the mass consisted of mature adipose tissue with fibrous septae and occasional thick-walled vessels with prominent smooth muscle bundles. A rare atypical stromal cell was present, otherwise no significant cytologic atypia or lipoblasts were identified. After excluding fat-predominant angiomyolipoma and well-differentiated liposarcoma, a diagnosis of renal replacement lipomatosis was made. Renal replacement lipomatosis is a benign condition typically associated with a nonfunctioning or poorly functioning kidney often linked to renal calculus disease or chronic renal infection. The presentation in our case was atypical given an absence of associated renal calculus disease. This case is intended to increase awareness of this less commonly encountered entity as it may be confused with a fatty neoplasm of the kidney, some with malignant potential.
\end{abstract}

\section{Introduction}

Renal replacement lipomatosis of the kidney is a rare, benign condition characterized by marked atrophy of renal parenchyma with fibrofatty proliferation of the renal sinus and is often associated with long-standing inflammation [1] and renal calculi in $76-79 \%$ of cases $[2,3]$. Rare cases are idiopathic. In our report, we present a case of renal replacement lipomatosis not associated with renal calculus disease. The differential diagnosis and key imaging findings that served to establish this specific diagnosis with histopathologic confirmation are reviewed.

\section{Case Presentation}

A 51-year-old woman with a past medical history of pancreas transplant for type 1 diabetes subsequently developed ureteral stricture. This was initially managed by a nephrostomy tube and nephroureterostomy stenting with periodic exchanges every two months to help restore urine flow; however, the renal function of the kidney progressively declined with recurrent and complicated urinary tract infections. She presented for kidney transplant with right native nephrectomy. Additionally, the patient has a history of left native nephrectomy at age 2 but the reason was not 


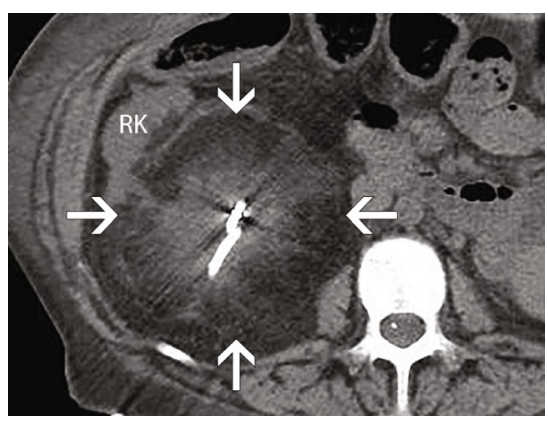

(a)

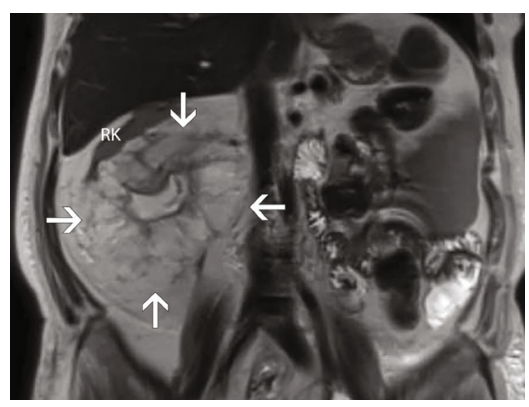

(b)

FIGURE 1: Imaging studies of the right kidney. (a) CT of the abdomen without contrast, axial view. At the level of the mass (outlined by white arrows), the right kidney (RK) is atrophic and there is extensive lipomatous proliferation of the renal sinus fat. The bright linear structure in the middle of the mass is a percutaneous drainage tube. (b) MRI of the abdomen without fat suppression or contrast, coronal T2- weighted image. There is a prominent hyperintense fatty proliferation of the renal sinus fat (outlined by white arrows) displacing the right kidney (RK). There is an associated thin rim of enhancing adjacent renal parenchyma.

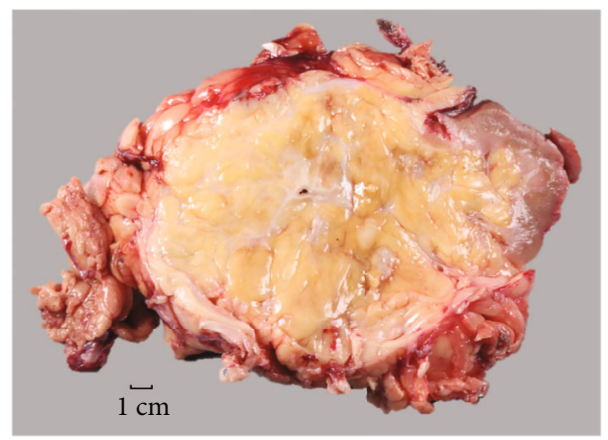

FIGURE 2: Gross photograph of bisected right kidney. The kidney is largely replaced by an extensive fatty proliferation of the renal sinus fat. There is adjacent surrounding residual compressed red-brown renal parenchyma.

clearly documented. There was no previous history of renal calculi. Preoperative abdominal CT and MRI showed an atrophic right kidney with extensive lipomatous proliferation of the renal sinus fat (Figures 1(a) and 1(b)). There was extensive inflammation within the collecting system and a percutaneous drainage tube was present. On gross examination, a markedly distorted nephrectomy specimen was received with a moderate amount of attached perinephric fat, with a weight of 1,040 grams and overall dimensions of $19.7 \times 12.7 \times 10.4 \mathrm{~cm}$. The cut surface revealed a $14.7 \times$ $12.8 \times 12.2 \mathrm{~cm}$ gray-yellow to white, lobulated, renal sinus lipomatous mass with fibrous septae replacing approximately $85 \%$ of the kidney (Figure 2). Areas of hemorrhage and degeneration were also noted. The residual renal parenchyma was red-brown and unremarkable. Microscopic examination revealed the mass consisted of mature adipose tissue with fibrous septae and occasional thick-walled vessels with prominent smooth muscle bundles. A rare atypical stromal cell was present, otherwise no significant cytologic atypia or lipoblasts were identified. There was a clear demarcation between adipose tissue and residual renal parenchyma at the periphery (Figures 3(a)-3(d)), which showed relatively mild chronic changes. Arteries showed fibrous intimal thick- ening. Mild patchy interstitial fibrosis and tubular atrophy were estimated to be less than $25 \%$. Glomerular obsolescence was approximately $20 \%$. Mild mesangial matrix expansion without well-developed Kimmelstiel-Wilson nodules by PAS stain was seen. Immunohistochemical studies with antibodies against HMB-45, Melan-A, and SMA did not support a diagnosis of renal angiomyolipoma. Immunohistochemistry and fluorescence in situ hybridization to detect MDM2 expression and $M D M 2$ amplification, respectively, were negative. After excluding a fat-predominant angiomyolipoma and well-differentiated liposarcoma, a diagnosis of renal lipomatosis was made. The patient remained asymptomatic after 8 months of follow-up.

\section{Discussion}

Renal replacement lipomatosis has been described using a variety of terms including renal fibrolipomatosis $[4,5]$, renal replacement lipomatosis of the kidney [2, 6, 7], fatty transformation of the kidney [8], fatty replacement of the kidney, lipomatous paranephritis, and lipoma diffusum renis [9]; however, the term renal replacement lipomatosis is most used at present. The first case was described in 1841 by 


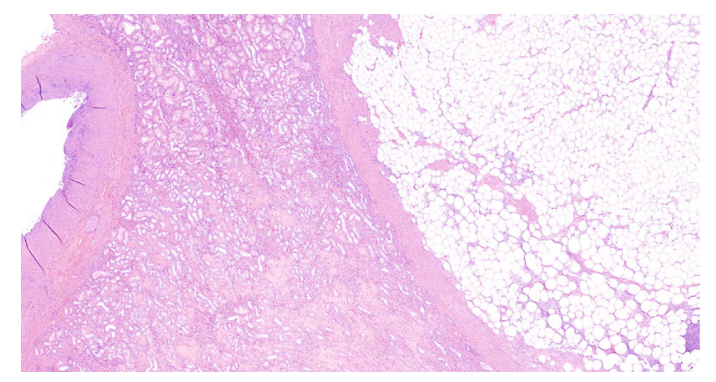

(a)

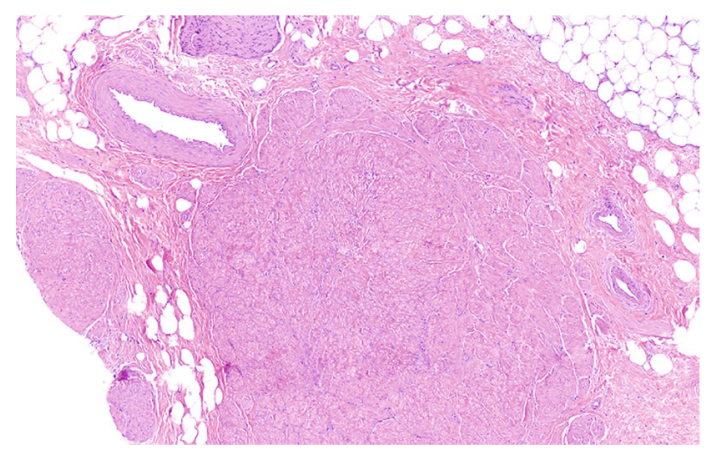

(c)

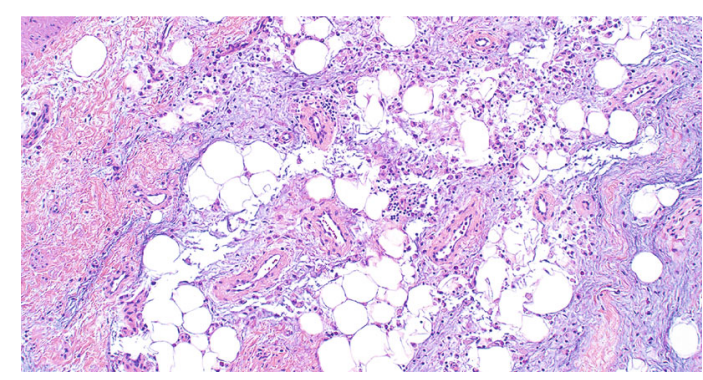

(b)

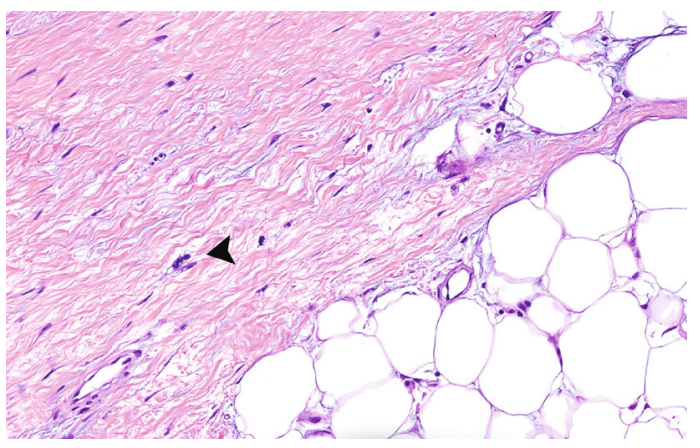

(d)

Figure 3: Microphotograph of the right kidney. (a) Well-demarcated mature adipose tissue and adjacent renal parenchyma. (b) Renal sinus fat showing inflammatory changes with areas of hyalinization and fat necrosis. (c) The lipomatous proliferation of the renal sinus fat contains fibrous septae and occasional thick-walled vessels with prominent smooth muscle bundles. (d) Rare atypical stromal cell (arrowhead), otherwise no significant cytologic atypia or lipoblasts were identified.

Rayer [10] occurring in an autopsy performed in 1837 [11]. Several reported cases have been reported in the English literature, the majority of which are individual case reports and a few large case series [2, 3, 12]. Renal replacement lipomatosis is a rare, benign condition characterized by prominent fat proliferation of the renal sinus with atrophy of the renal parenchyma that may mimic other renal malignancies. It typically occurs unilaterally, but bilateral cases have been reported [13] and are thought to result in association with infection, obstructive renal calculi, or long-standing hydronephrosis. Rare cases are idiopathic [13-15]. A major differential diagnosis for infection, often associated with chronic obstruction from calculi, is xanthogranulomatous pyelonephritis (XGP). XGP is characterized by destruction of the renal parenchyma and replacement by lipid-laden macrophages, whereas renal lipomatosis is a fatty proliferation of the renal sinus with atrophy of the renal parenchyma [16-18]. Cases of XGP coexisting with renal lipomatosis have been reported [19-22] as well as with malignancy [23].

Many of the tumors of the renal pelvis include urothelial carcinoma, with squamous cell carcinoma accounting for a smaller percentage of these cases. Renal parenchymal tumors such as renal cell carcinoma commonly extend into the renal sinus. Rarely, tumors originating from mesenchymal tissue develop in the renal sinus. Renal lipomatosis may be confused with fat-containing renal tumors such as renal lipoma, angiomyolipoma, or liposarcoma. In contrast to renal lipomatosis, these tumors are generally located in intrarenal or extrarenal areas outside the renal sinus [24, 25]. Renal lipomas tend to be small and originate in the fat cells within the renal capsule. Renal liposarcomas arise peripherally in perirenal fat within Gerota's fascia or within the renal capsule and may distort the kidney but usually do not invade the adjacent renal parenchyma. Patients with lipoma, angiomyolipoma, or liposarcoma also tend to have no other associated pathology except in compressed areas immediately surrounding the tumor without impact on overall renal function [26], whereas with renal lipomatosis there is evidence of infection and displacement of normal renal structures by fat. Angiomyolipoma and liposarcoma can be easily differentiated from renal lipomatosis. The presence of smooth muscle fibers, thick-walled blood vessels, and coexpression of melanocytic and smooth muscle markers by immunohistochemistry in myoid and lipoid components in angiomyolipoma and atypical stromal cells with detection of MDM2 expression by immunohistochemistry and genetic amplification by fluorescence in situ hybridization is absent in renal lipomatosis.

In conclusion, renal lipomatosis may be confused with lipomatous neoplasms of the kidney. It is usually associated with a unilateral nonfunctioning or poorly functioning kidney. When present in a native kidney that is deemed nonfunctional, nephrectomy is often adequate treatment [17]. We want to raise awareness among pathologists of this entity, as it is infrequently encountered and may be difficult to differentiate from other fat-containing tumors in the renal sinus preoperatively. 


\section{Conflicts of Interest}

The authors do not have a conflict of interest associated with this publication.

\section{Authors' Contributions}

$\mathrm{KC}$ served as the primary author and is responsible for this literature review and construction of the manuscript. EB and $\mathrm{KC}$ were involved in the gross examination and handling of the surgical specimen. KC, LW, TT, and MH have been involved in the requisition and analysis of the data. $\mathrm{MH}$ served as the senior author managing the construction and edits of the manuscript and guiding the primary author through the submission process. All authors read, revised, and gave approval of the manuscript.

\section{Acknowledgments}

The authors would like to thank Dr. William C. Goggins (Transplant Surgery, Indiana University, Indianapolis, IN), Dr. Karen Rea (Radiology, Indiana University, Indianapolis, IN), and Dr. Carrie L. Phillips (Renal Pathology, Indiana University, Indianapolis, IN) for their contributions to this case report. The skillful digital image preparation by Fredrik Skarstedt is gratefully acknowledged. This work was supported by Indiana University, Department of Pathology and Laboratory Medicine, Indianapolis, Indiana.

\section{References}

[1] M. A. Ambos, M. A. Bosniak, R. Gordon, and M. A. Madayag, "Renal replacement lipomatosis of the kidney," American Journal Roentgenology, vol. 130, no. 6, pp. 1087-1091, 1978.

[2] A. A. Kutzmann, "Renal lipomatosis of kidney," Surgery, Gynecology \& Obstetrics, vol. 52, pp. 690-701, 1931.

[3] L. J. Roth and H. B. Davidson, "Fibrous and fatty replacement of renal parenchyma," Journal of the American Medical Association, vol. 111, no. 3, pp. 233-239, 1962.

[4] O. Olson and P. Weiland, "Renal fibrolipomatosis," Acta Radiologica: Diagnosis, vol. 1, no. 5, pp. 1061-1070, 1963.

[5] L. Kreel and R. N. Memed, "Renal fibrolipomatosis," The British Journal of Radiology, vol. 39, no. 467, pp. 837-843, 1966.

[6] A. H. Peacock and A. Balle, "Renal lipomatosis," Annals of Surgery, vol. 103, no. 3, pp. 395-401, 1936.

[7] J. B. Priestley, "Renal lipomatosis or fatty replacement of destroyed renal cortex," The Journal of Urology, vol. 40, no. 2, pp. 269-275, 1938.

[8] E. Rickards, "Remarks on the fatty transformation of the kidney," British Medical Journal, vol. 2, no. 1175, pp. 2-3, 1938.

[9] D. Faegenburg, M. Bosniak, and J. A. Evans, "Renal sinus lipomatosis: its demonstration by nephrotomography," Radiology, vol. 83, no. 6, pp. 987-998, 1964.

[10] P. Rayer, "Traité des Maladies des Reins Et des Altérations de la Sécrétion Urinaire," in Étudiées en Elles-Mêmes Et dans Leurs Rapports Avec les Maladies des Uretères de la Vessie, de la Prostate, de l'Urètre Etc, vol. 3, (French edition), p. 614, Avec un Atlas In-Folio, 2001.
[11] H. L. Gildenhorn, "Renal replacement lipomatosis," Journal of the American Medical Association, vol. 181, no. 11, pp. 994997, 1962.

[12] J. N. Poilly, J. E. Dickie, and W. B. James, "Renal sinus lipomatosis: a report of twenty- six cases," British Journal of Urology, vol. 41, no. 3, pp. 257-266, 1969.

[13] N. H. Setty, K. Uma, V. N. Narvekar, and R. S. Desai, "Bilateral idiopathic replacement lipomatosis of the kidney with posterior mediastinum lipomatosis," Indian Journal of Radiology and Imaging, vol. 12, no. 2, pp. 251-252, 2002.

[14] V. B. Shah, A. B. Rupani, M. S. Deokar, and H. R. Pathak, "Idiopathic renal replacement lipomatosis: a case report and review of literature," Indian Journal of Pathology \& Microbiology, vol. 52, no. 4, pp. 552-553, 2009.

[15] G. Bhat, V. Barude, S. Anuradha, V. Tembadamani, and S. Hedge, "Idiopathic renal replacement lipomatosis: a diagnostic and therapeutic challenge," Turkish Journal of Surgery, vol. 34, no. 3, pp. 250-252, 2018.

[16] N. A. Choh, M. Jehangir, and S. A. Choh, "Renal replacement lipomatosis: a rare type of renal pseudotumor," Indian Journal of Nephrology, vol. 20, no. 2, pp. 92-93, 2010.

[17] M. Khan, S. S. Nazir, S. Ahangar, S. J. F. Qadri, and N. A. Alroo, "Total renal replacement lipomatosis," International Journal of Surgery, vol. 8, no. 4, pp. 263-265, 2010.

[18] S. M. Ando, R. A. Moreno, P. C. C. Viana, and F. I. Yamauchi, "Extensive renal sinus lipomatosis in xanthogranulomatous pyelonephritis simulating liposarcoma," International Brazilian Journal of Urology, vol. 44, no. 3, pp. 642-644, 2018.

[19] B. Acunas, G. Acunas, I. Rozanes, N. Buyukbabani, and E. Gokmen, "Coexistent xanthogranulomatous pyelonephritis and massive replacement lipomatosis of the kidney: CT diagnosis," Urologic Radiology, vol. 12, no. 1, pp. 88-90, 1990.

[20] K. K. Prasad, R. Pandey, M. Kathuria, and P. K. Pradhan, "Co-existent massive renal replacement lipomatosis and xanthogranulomatous pyelonephritis - a case report," Indian Journal of Pathology \& Microbiology, vol. 46, no. 4, pp. 674675, 2003.

[21] Y. Sakata, N. Kinoshita, H. Kato, Y. Yamada, and Y. Sugimura, "Coexistence of renal replacement lipomatosis with xanthogranulomatous pyelonephritis," International Journal of Urology, vol. 11, no. 1, pp. 44-46, 2004.

[22] A. Kiris, E. Kocakoc, A. K. Poyraz, F. Dagli, and Y. Boztosun, "Xanthogranulomatous pyelonephritis with nephrocutanous fistula and coexisting renal replacement lipomatosis: the report of a rare case," Clinical Imaging, vol. 29, no. 5, pp. 356-358, 2005.

[23] P. Arora, S. Rao, N. Khurana, and V. K. Ramteke, "Renal replacement lipomatosis with coexistent papillary renal cell carcinoma, renal tubulopapillary adenomatosis and xanthogranulomatous pyelonephritis: an extremely rare association and possible pathogenetic correlation," Urology Journal, vol. 10, no. 2, pp. 906-908, 2013.

[24] C. Yağci, P. Koşucu, M. Yöröübulut et al., "Renal replacement lipomatosis: ultrasonography and computed tomography findings," European Radiology, vol. 9, no. 8, pp. 1599-1601, 1999.

[25] S. Karasick and R. J. Wechsler, "Case 23: Replacement lipomatosis of the kidney," Radiology, vol. 215, no. 3, pp. 754-756, 2000.

[26] S. D. Chang, F. V. Coakley, and R. B. Goldstein, "Renal replacement lipomatosis associated with renal transplantation," The British Journal of Radiology, vol. 78, no. 925, pp. 60-61, 2005. 